\title{
Platinum-Resistant Ovarian Carcinoma
}

National Cancer Institute

\section{Source}

National Cancer Institute. Platinum-Resistant Ovarian Carcinoma. NCI Thesaurus. Code C147561.

Ovarian carcinoma that is resistant to platinum therapy. 